\title{
The study of energy balance in metals under deformation and failure in application to fatigue crack propagation process
}

\author{
By A. Iziumova, A. Vshivkov, A. Prokhorov, A. Kostina and O. Plekhov
}

Institute of continuous media mechanics UB RAS, 1, Ac. Koroleva str., 614013, Perm, Russia, poa@icmm.ru

\begin{abstract}
The real time temperature measurement allows us to carry out the study of energy balance in metals under quasi-static loading and fatigue crack propagation. The temperature field near the crack tip was recorded by infrared camera FLIR SC5000. The heat source at the crack tip was calculated based on temperature data treatment and verified based on the original heat flow detector. The two regimes of energy dissipation at crack tip during its propagation in Paris regime were shown. The experimental results were supported by numerical simulation. The original theoretical model was adapted for standard final-elements numerical code (Abaqus) to simulate energy dissipation under quasi-static and cyclic loading in 3D geometries.
\end{abstract}

\section{Introduction}

It is now well known that the real materials have a complex structure which can be considered as a hierarchy of the different scale levels. Structural changes occur in all scale levels and lead to irreversible deformation and failure of the material. The development of the irreversible deformation is accompanied by the processes of energy dissipation and storage and, as a result, leads to the changing of material temperature (self heating). From the thermodynamical point of view we can conclude that the expended mechanical energy is converted into thermal energy generated by movement and annihilation of the defects and stored energy accumulated in the elastic fields of the defects. These process can be observed both in the whole sample volume (under quasi-static loading) and locally (in crack tip under crack propagation).

The process of fatigue crack propagation in metals is accompanied by the formation of monotonic and cyclic plastic deformation zones at a crack tip and, as a consequence, rising up the temperature at the crack tip. The generally used small scale yielding assumption allows one to apply the results of linear fracture mechanics (stress intensity factor) in order to describe the fatigue crack growth. The power relation between stress intensity factor and crack rate was called Paris law. The effectiveness of this law and its extensive use in engineering practice does not eliminate the problem of searching for new kinetic relations for cracks that take into account the physics of the processes accompanying changes in the material structure at crack tip. Many authors [1-3] have proposed alternative function relating the crack rate and such values as J-integral, plastic deformation zone size, the value of dissipated energy, etc. The use of the energy approach in the description of fatigue crack propagation originates in [4-9]. One of the most detailed descriptions of energy balance at the fatigue crack tip was proposed in [9]. A similar relation was obtained in [10] where the law of crack propagation was derived as entropy production in the system written as product of driving force responsible for the translational mode on the corresponding thermodynamic flow. In [11], the authors experimentally demonstrated the relationship between the work of plastic deformation and the change in the stress intensity factor. Based on the assumption that most part of this work is converted into heat they derived the fatigue crack propagation law.

The construction of energy based crack propagation law requires the development of a theoretical description of energy balance in metals under deformation and failure and experimental technique for measurement of energy dissipation rate suitable for both laboratory conditions and for real engineering structures. The infrared thermography gives us a unique possibility to measure the temperature filed on a sample surface with high time and spatial resolution. The data treatment algorithm including noise filtering for temperature measurements based on infrared radiation data, compensation of the external conditions of the experiment (reflection of infrared waves from the metal specimen surface, relative motion of a specimen, etc.), and calculation of heat losses in the course of the experiment was proposed in [12]. These algorithms allow us to calculate the heat sources in the sample during deformation and failure. To increase the reliability of heat sources calculation we develop additional heat measuring setup. The setup is based on the Seebeck effect and includes two Pelietier elements and temperature controlling feedback. The two different techniques provide a high reliability of heat sources measurement. These experiments allow us to study in detail a dissipated and stored energy evolution in metals (AISI304, titanium OT-4(Rus)) and support the conception originally formulated by $\mathrm{J}$. H. Lambert in 1779 about energy similarity of mechanical and thermal failure processes of solids. The essence of this similarity is that the mechanical failure of metals can occur when the relative deformation in at least one direction reaches a value equal to the linear thermal expansion at the melting point. A substantial contribution to the development of these ideas was made by V.S. Ivanova, who proposed the structure-energy theory of metal fracture [13]. A detailed analysis of thermodynamic effects produced by cyclic deformation and failure in metals was carried out by V.T. Troshchenko and V.V. Fedorov. The overview of strength energy criteria was published in $[14,15]$. 
The most intriguing result is that the classical Paris`s regime of crack propagation can be divided in two regimes with different kinetics of energy dissipation. During the first regime the crack rate is linear function of crack length and power of heat dissipation $\left(\frac{\partial a_{1}}{\partial N} \sim Q_{1} a_{1}\right)$. The second regime is characterized by generally expected linear relation between crack rate and power of heat dissipation $\left(\frac{\partial a_{2}}{\partial N} \sim Q_{2}\right)$.

The experimental results give impetus to the theoretical description of energy balance in metal. One of the possible ways of the stored energy estimation is the use of stress-strain curve [16,17]. In [18], it is shown that this method can give us only part of the stored energy value connected with non-homogeneous plastic deformation. In [19], the stored energy is calculated with the use of the discrete dislocation plasticity model which allows one to calculate the value of the stored energy directly as the difference between internal energy associated with the dislocation in the current state and in the initial. The internal variables approach is used in the works $[20,21]$. The authors suggest that the energy connected with plastic deformation is a function of internal variables only.

The analysis of the existing models lets us assume that the stored energy in the material occurs due to the evolution of defects of different structural levels and for its adequate description it is necessary to obtain constitutive equations of the evolution of structural defects. In the extension of our experimental results we propose the development of a statistical model of the defect evolution [22]. Evolution of the mesodefect ensembles is modelled with the use of the internal field variable which can be considered as a defective induced deformation. The developed model was adapted for using in standard finite-elements numerical code (Abaqus) to simulate energy dissipation under quasi-static and cyclic loading in 3D geometries. The efficiency of the obtained equations is demonstrated by the simulation of the energy balance in AISI304 steel under quasi-static deformation and crack propagation.

\section{Experimental study of crack propagation in metals}

\subsection{Experimental setup}

Experimental study of temperature evolution was carried out on the plane samples of titanium alloy OT-4 (Russian marking) and stainless steel AISI304. The sample geometries are presented in figure 1. All sizes are presented in millimetres. The fatigue experiments were carried out in collaboration with University of the Federal Armed Forces (Munich, Germany) and HИO-2, laboratory «Physics of strength and smart diagnostic system» Tolyatti state university (Tolyatti, Russia) in the frequency range from 10 to $20 \mathrm{~Hz}$. The cycle asymmetry coefficient was equals 0.1 and -1 for samples presented in figure 1.a and 1.b, respectively. The surfaces of the specimens were polished in several stages by the abrasive paper (at the final stage of polishing the grit size does not exceed $3 \mu \mathrm{m}$ ). Before starting the experiment the polished surface was covered by a thin layer of amorphous carbon.

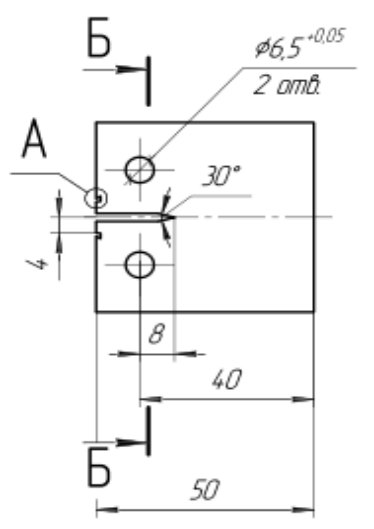

a)

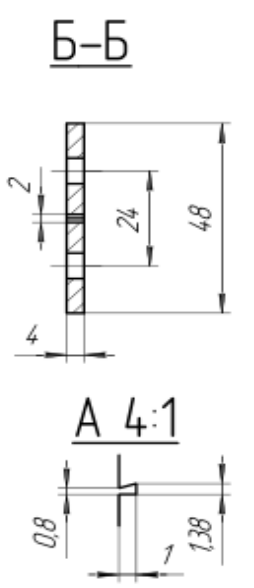

)

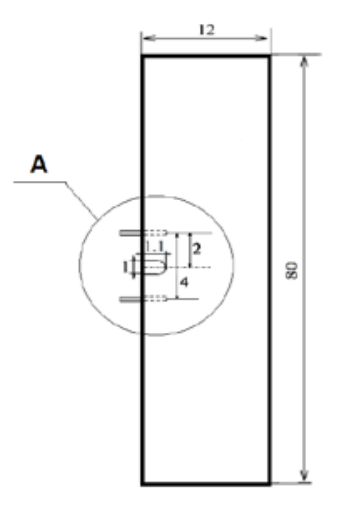

Fig 1. Specimen's geometry
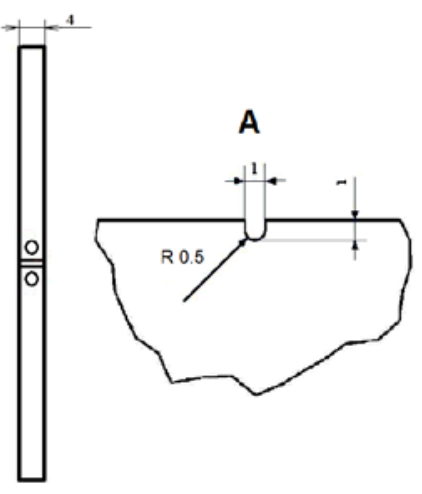

b)

The temperature evolution was recorded by infrared camera FLIR SC 5000 . The spectral range of the camera is 3-5 $\mu \mathrm{m}$. The maximum frame size is $320 \times 256$ pixels; the spatial resolution is $10^{-4}$ meters. The temperature sensitivity is $25 \mathrm{mK}$ at $300 \mathrm{~K}$. Calibration of the camera was made based on the standard calibration table. It was used FLIR SC5000 MW G1 F/3.0 close-up lens (distortion is less than $0.5 \%$ ) to investigate the plastic zone in details.

To measure the heat flow near the crack tip the original Peltier-based experimental setup was developed. To control the crack length an original measurement system has been developed. The crack length measurement system is based on the potential drop technique. The principal scheme of experiments is presented in figure 2. The following notations are accepted in figure 2: Testing sample (1); Infrared camera (2); Peltier element (3); Potential drop measuring setup (4); Analog amplifier MDU-8 (5); Analog digital converter ADC NI USB-6251 (6); PC with LabView software (7). 


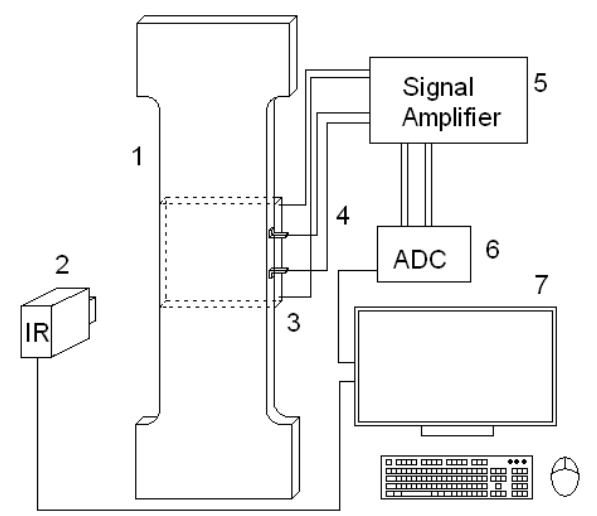

Fig. 2 Principal scheme of experimental setup.

\subsection{Experimental results}

Figure 3 presents the characteristic results of the experiments with constant stress amplitude. The evolutions of heat source at crack tip and crack rate versus time of the experiment for different stress amplitudes are presented in figure 3.a and 3.b, respectively. The cracks are propagated in the Paris regime - figure 3.c presents the power function between crack rate and stress intensity factor range for both stress amplitudes.

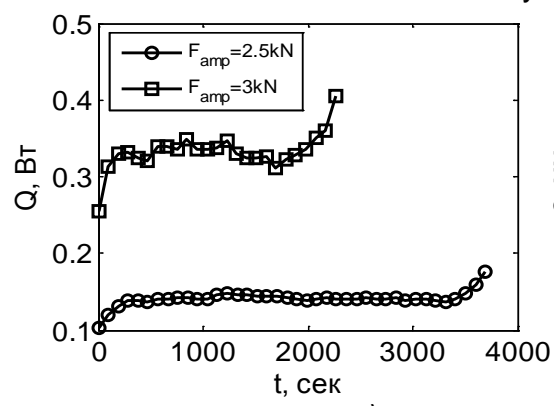

a)

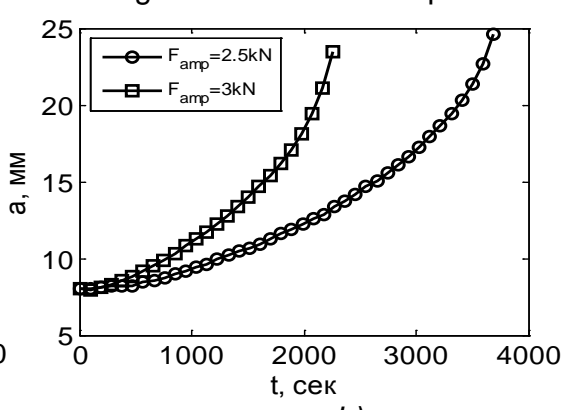

b)

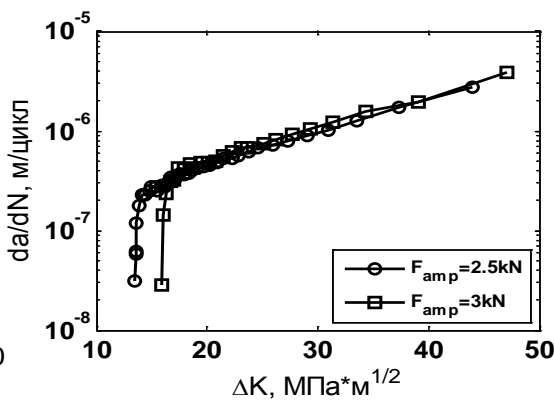

c)

Fig. 3 a-evolution of heat source at crack tip versus time of the experiment; $b$-crack length versus time of the experiment, $c$ - crack rate versus stress intensity factor range.

During the first part of the experiment $(\approx 0-250$ second for stress amplitude $2,5 \mathrm{kH}$ and $\approx 0-100$ second for stress amplitude $3 \mathrm{kH}$ ) the measured heat flux rises to the constant value. The crack length is constant during this period. The following period of the experiment can be divided into two parts. The first part from 250 to 3300 second for stress amplitude 2,5kH and from 100 to 1900 second for stress amplitude $3 \mathrm{kH}$ corresponds to constant value of heat flux. The last part is characterized by rise of heat flux. This part of the experiment is finished by critical, unstable regime of crack propagation.

The analysis of experimental data was carried separately for each regime of heat dissipation. Figure 4 presents the two aforementioned regime of crack propagation in different coordinates.

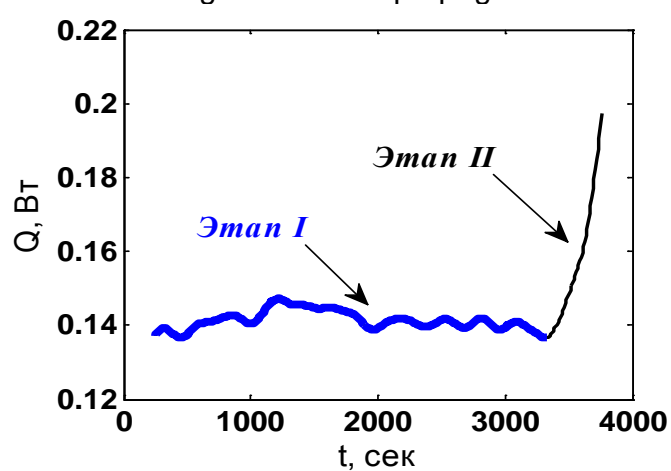

a)

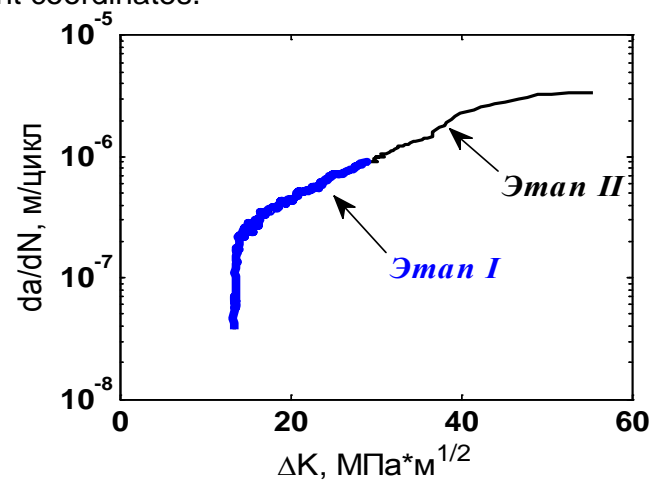

b)

Fig. 4 Two regimes of heat dissipation under crack propagation a - in the plot of heat dissipation rate versus time $b$ - in the plot of crack rate versus stress intensity factor range. 
The figure 5 presents the evolution of crack rate versus relative heat flux and crack length. The relative value of heat flux and relative product of crack length on heat flux was determined as follows

$$
Q_{1} a_{1}^{\prime}=\frac{Q_{1} a_{1}-Q_{1} a_{1}^{\min }}{Q_{1} a_{1}^{\max }-Q_{1} a_{1}^{\min }}, Q_{2}^{\prime}=\frac{Q_{2}-Q_{2}^{\min }}{Q_{2}^{\max }-Q_{2}^{\min }} .
$$

The results presented in figure 5 exhibit the linear relation for both regimes of crack propagation. The classical Paris`s regime of crack propagation can be divided into two regimes with different kinetics of energy dissipation. During first regime the crack rate is linear function of crack length and power of heat dissipation $\left(\frac{\partial a_{1}}{\partial N} \sim Q_{1} a_{1}\right)$. The second regime is characterized by generally expected linear relation between crack rate and power of heat dissipation $\left(\frac{\partial a_{2}}{\partial N} \sim Q_{2}\right)$

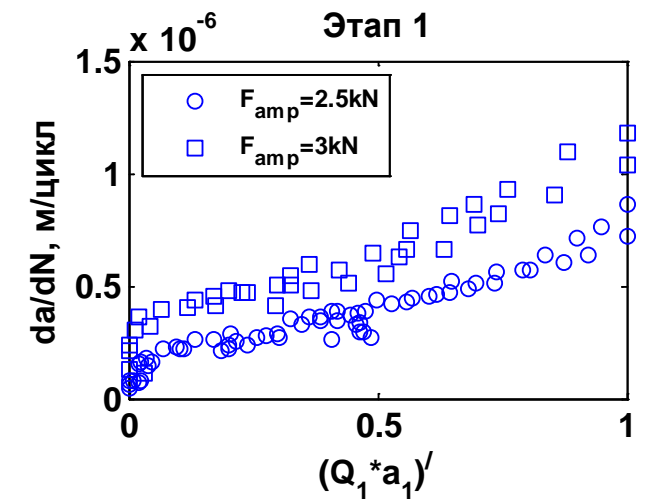

a)

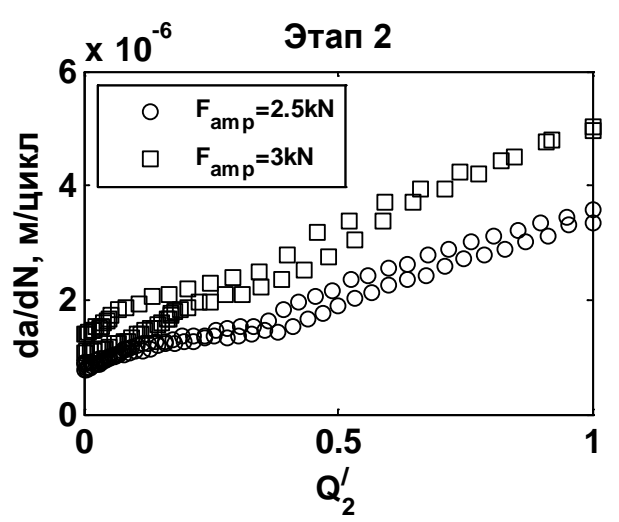

b)

Fig. 5 The evolution of crack rate versus relative crack length multiplied by heat flux from the crack tip (a) and crack rate versus relative heat flux from the crack tip (b).

\section{Theoretical study on energy dissipation in metals}

\subsection{Mathematical model energy balance in metals with defects}

The understanding of physical nature of temperature rise under deformation and failure and a development monitoring technique and energy based fracture criteria request the development of mathematical model of defect evolution in metals. One of the possible ways to construct such model is the development of statistical description of mesodefect ensemble proposed in [22]. Typical mesoscopic defects (microshears) are introduced as a symmetrical second order tensor $\tilde{s}$

$$
\tilde{s}=1 / 2(\vec{n} \vec{b}+\vec{b} \vec{n})
$$

where $\vec{n}$ - a unit normal to the shear plane, $\vec{b}$ - a unit vector in shear direction, $S$ - shear intensity.

Macroscopic tensor of the defect density which has a meaning of an additional (structural) strain can be calculated by the averaging of $\tilde{s}$ over an elementary volume:

$$
\tilde{p}=n\langle\widetilde{s}\rangle
$$

where $n$ is the number of defects per unit volume.

The defect distribution function can be written as:

$$
W=Z^{-1} \exp (-E / \Theta)
$$

where $E$ - the energy of the defect, $Z$ - normalizing factor, $\Theta$ - the effective temperature factor.

Energy of a defect up to a constant was written in the framework of mean field approximation [22]. It leads to the self-consistency equation for the determination of the defect density tensor 


$$
\tilde{p}=n \int \tilde{s} W(\tilde{s}) d \tilde{s}
$$

Solution of (5) for the case of pure shear is presented in [22]. Based on these results we can formulate a model which describes the defect evolution and the energy balance in a material. Let us assume that the full strain rate $\dot{\widetilde{\varepsilon}}$ can be represented as the sum of three components: elastic $\left(\dot{\vec{\varepsilon}}^{e}\right)$, plastic $\left(\dot{\widetilde{\varepsilon}}^{p}\right)$ and structural $(\dot{\vec{p}})$ :

$$
\dot{\widetilde{\varepsilon}}=\dot{\widetilde{\varepsilon}}^{e}+\dot{\widetilde{\varepsilon}}^{p}+\dot{\vec{p}}
$$

Elastic strains are obeyed to the Hook's law. Inelastic part can be described based on the thermodynamic laws, we can obtain the dissipation inequality in the following form:

$$
\tilde{\sigma}: \dot{\vec{\varepsilon}}-\rho(\dot{F}+\dot{T} S)-\vec{q} \nabla T / T \geq 0
$$

where $F=e-T S$ - a free energy, $e$ - an internal energy, $T$ - temperature, $S$ - entropy, $\vec{q}$ - heat flux vector.

The main assumption of the approach is a function of free energy which is written as:

$$
F=F\left(T, \widetilde{\varepsilon}^{e}, \tilde{p}\right)
$$

Based on this assumption the dissipation can be written as follows

$$
\tilde{\sigma}: \dot{\widetilde{\varepsilon}}^{p}+\left(\tilde{\sigma}-\rho \frac{\partial F}{\partial \widetilde{p}}\right): \dot{\vec{p}}-\vec{q} \nabla T / T \geq 0
$$

According to the Onsager's principle we can obtain quasi linear relations for the thermodynamic forces and flows:

$$
\begin{aligned}
& \dot{\dot{\varepsilon}}^{p}=L_{\sigma} \tilde{\sigma}+L_{p \sigma}\left(\tilde{\sigma}-\rho \frac{\partial F}{\partial \tilde{p}}\right), \\
& \dot{\tilde{p}}=L_{\sigma p} \tilde{\sigma}+L_{p \sigma}\left(\tilde{\sigma}-\rho \frac{\partial F}{\partial \widetilde{p}}\right) .
\end{aligned}
$$

where $L_{\sigma}, L_{p \sigma}, L_{p}$ - kinetic coefficients.

Thermodynamic force $\left(\tilde{\sigma}-\rho \frac{\partial F}{\partial \tilde{p}}\right)$ can be determined as:

$$
\left(\tilde{\sigma}-\rho \frac{\partial F}{\partial \tilde{p}}\right)=\frac{\tilde{\sigma}}{2 G \sigma_{c}}-f\left(\frac{|p|}{p_{c}}\right) \frac{\tilde{p}}{p_{c}}
$$

where $\sigma_{c}, p_{c}$ - scale factors, $|p|$ - intensity of the $\tilde{p}$ tensor. It is assumed that $f$ - is a power function of $|p|$ for modelling of nonlinear hardening:

$$
f\left(\frac{|p|}{p_{c}}\right)=k\left(\frac{|p|}{p_{c}}\right)^{\alpha}, k=\text { const }
$$

where $k$ is a scaling factor, $\alpha$ is an exponent.

Kinetic coefficients have the following form: 


$$
\begin{gathered}
L_{\sigma}=1 / \tau_{\sigma} \exp \left(-\frac{|\sigma|-\sigma_{c}}{a_{1}}\right), \\
L_{p}=1 / \tau_{p}\left[1+\exp \left(-\frac{\left.H\left(|\sigma|,|p|, \delta, p_{c}, \sigma_{c}\right)-\sigma_{y}\right)}{a_{2}}\right)\right], \\
L_{o p}=1 / \tau_{\text {op }},
\end{gathered}
$$

where $\tau_{\sigma}, \tau_{p \sigma}, \tau_{p}$ - characteristic relaxation times, $|\sigma|$ - stress tensor intensity, $\sigma_{y}$ - yield stress, $\sigma_{c}, a_{1}, a_{2}-$ material constants. The function $H\left(|\sigma|,|p|, \delta, p_{c}\right)$ can be considered as the "degree of the system nonequilibrium":

$$
H\left(|\sigma|,|p|, \delta, p_{c}\right)=|\sigma|-2 G \sigma_{c} f\left(|p| / p_{c}\right)
$$

The first thermodynamic law can be written as:

$$
\rho \dot{e}=\tilde{\sigma}: \dot{\widetilde{\varepsilon}}+\nabla \cdot \vec{q}+r
$$

where $r$ - heat supply.

Standard thermodynamic formalism give us an ter

$$
\rho c \dot{T}=\nabla \cdot \vec{q}+r+Q_{e}+Q_{p}
$$

where $Q_{e}=T \frac{\partial \sigma}{\partial T}: \dot{\widetilde{\varepsilon}}^{e}$ - the heating due to the thermoelastic effects, $Q_{p}=\tilde{\sigma}:\left(\dot{\widetilde{\varepsilon}}^{p}+\dot{\tilde{p}}\right)-\left[\rho \frac{\partial F}{\partial \tilde{p}}: \dot{\tilde{p}}-\rho T \frac{\partial^{2} F}{\partial T \partial \tilde{p}}: \dot{\tilde{p}}\right]-$ the inelastic contribution to the heating, $c$ - specific heat.

The energy balance equation has a term associated with the heat generation due to reversible deformation and the term corresponding to the heat generation due to irreversible deformation. Part of the plastic work is converted into the heat. The remaining part is stored in the material.

The rate of plastic work can be introduced as:

$$
\dot{W}^{p}=\tilde{\sigma}:\left(\dot{\dot{\dot{\varepsilon}}}^{p}+\dot{\vec{p}}\right)
$$

The fraction of the plastic work rate converted into heating can be written as:

$$
\beta=Q_{p} / \dot{W}^{p}=1-\left[\rho \frac{\partial F}{\partial \widetilde{p}}: \dot{\vec{p}}-\rho T \frac{\partial^{2} F}{\partial T \partial \widetilde{p}}: \dot{\tilde{p}}\right] / \tilde{\sigma}:\left(\dot{\dot{\dot{\varepsilon}}}^{p}+\dot{\tilde{p}}\right)
$$

The $1-\beta$ parameter is the energy storage rate and the subject under investigation of this work.

\subsection{Results and discussion}

The efficiency of the aforementioned model is demonstrated by the simulation of the stress-strain evolution of sample made from 304(L) austenitic stainless steel and energy dissipation under crack propagation. The numerical modeling of crack propagation was carried out in the finite-element package Simulia Abaqus 6.13. All using modulus have corresponding licenses. Figure 6 presents a result of numerical simulation of quasi-static tension at strain rate $4.3 \cdot 10^{-3} \mathrm{~s}^{-1}$. The points present experimental results obtained in [23]. Theoretical stress-strain curve has a good agreement with the experimental data. 


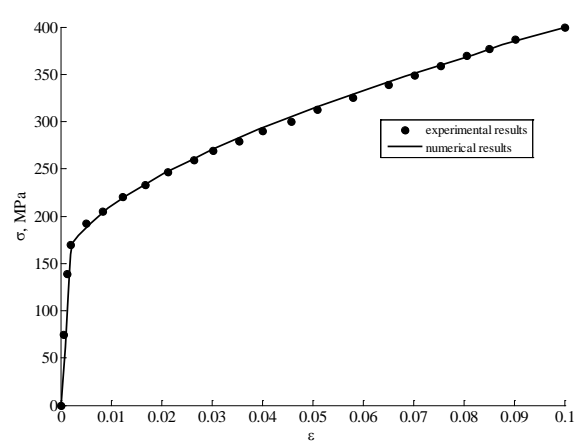

a)

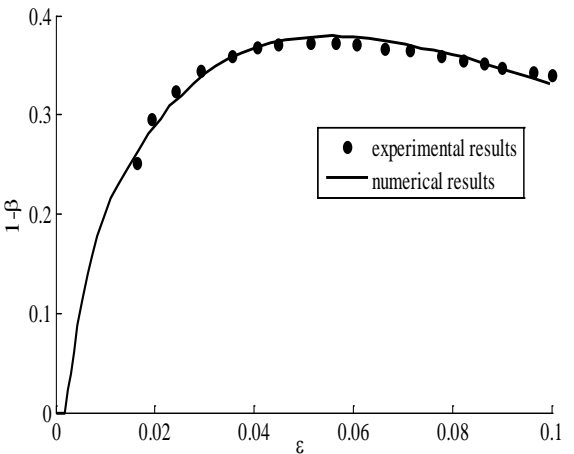

b)

Fig. 6 Stress-strain curve for the 304(L) austenitic stainless steel under quasistatic tension (a), corresponding stored energy evolution (b).

Calculated results of the energy storage rate connected with homogeneous plastic deformation are demonstrated in Fig. 6b. The obtained dependence has a maximum which agrees with the experimental data. According to the structural studies [23], on the initial stage of plastic deformation it is observed a preparation of the material structure associated with the initiation of new structural defects. This leads to a growth of the energy storage rate. When a strain reaches some level, the dominant processes are growth, movement and annihilation of structural defects. This leads to a growth of a dissipated energy portion.

The developed constitutive model allows us to investigate the correlation between the rate of strain hardening and energy storage rate. If the value of $L_{\sigma}$ exceeds the value of $L_{p}$ (predominantly dissipative plastic deformation without significant increase of the density of defects) the quasi-static diagram corresponds to the perfectly plastic material and the value of stored energy increases on the initial stage of plastic deformation and then it decreases to a constant (close to zero) value. For values of $L_{\sigma}<L_{p}$ (significant increase of the density of defects) the energy storage rate has only increasing segment of the curve and the stress-strain curve has a linear hardening.

The above explained model was used for simulation of crack propagation through the user subroutine UMAT. Arrays of material constants, strain, strain increments and the time step passed as the input data to the procedure. Increment of stress tensor components, increment of defect density tensor components and increment of a plastic strain are determined from the system of constitutive equations, which were represented as a finite difference scheme. Values of these components at the next time step are defined as the sum of values on the previous step and the appropriate increment.

The extended finite element method (XFEM) capability in Abaqus was used to model crack propagation. XFEM models a crack as an enriched feature by adding degrees of freedom in elements with special displacement functions. XFEM does not require the mesh to match the geometry of the discontinuities. It can be used to simulate initiation and propagation of a discrete crack along an arbitrary, solution - dependent path without the requirement of remeshing. To specify a fracture criterion based on the critical stored energy there was used a UDMGINI subroutine.

There is considered a three-dimensional problem of cyclic loading. Eight-node linear brick elements were used for the simulation. Figure 7 represents stresses corresponding to different time values of this cycle.

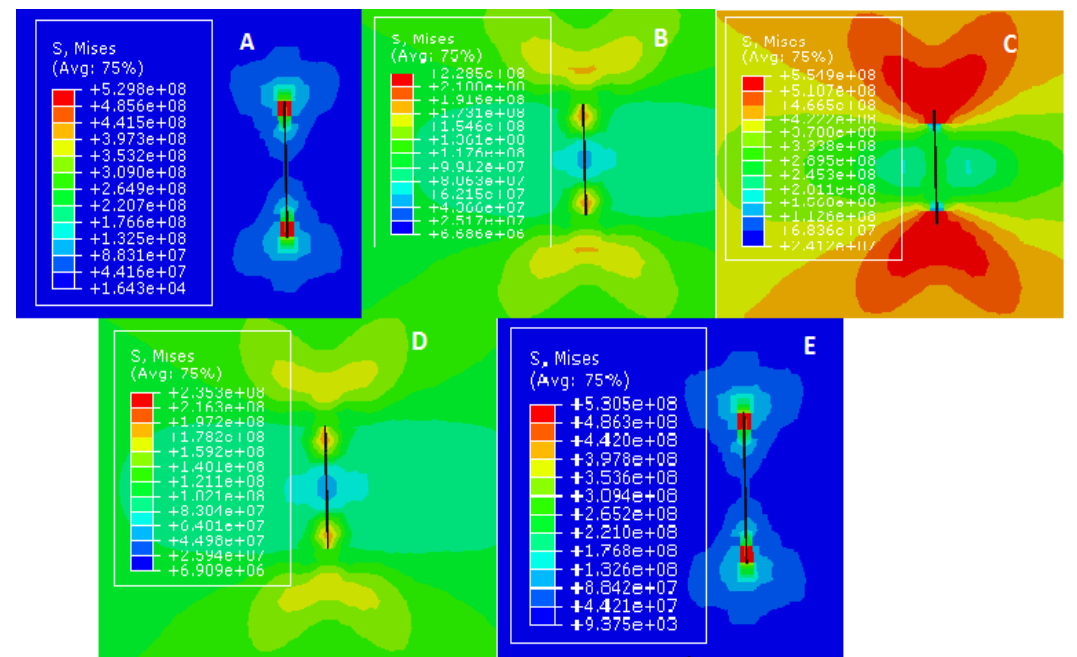

Fig. 7: Stress distributions in the crack tip corresponding to the $11^{\text {th }}$ cycle for different time moments: A) $t=5 \mathrm{c}, B$ ) $t=5.07$, C) $t=5.25, D) t=5.43, E) t=5.5$ 
Figure 8 presents information about the values of the stored energies and inelastic contribution to the heat generation at the different number of cycles. The crack starts to propagate when the value of the stored energy reaches $1.6 \cdot 10^{6} \mathrm{~J}$.

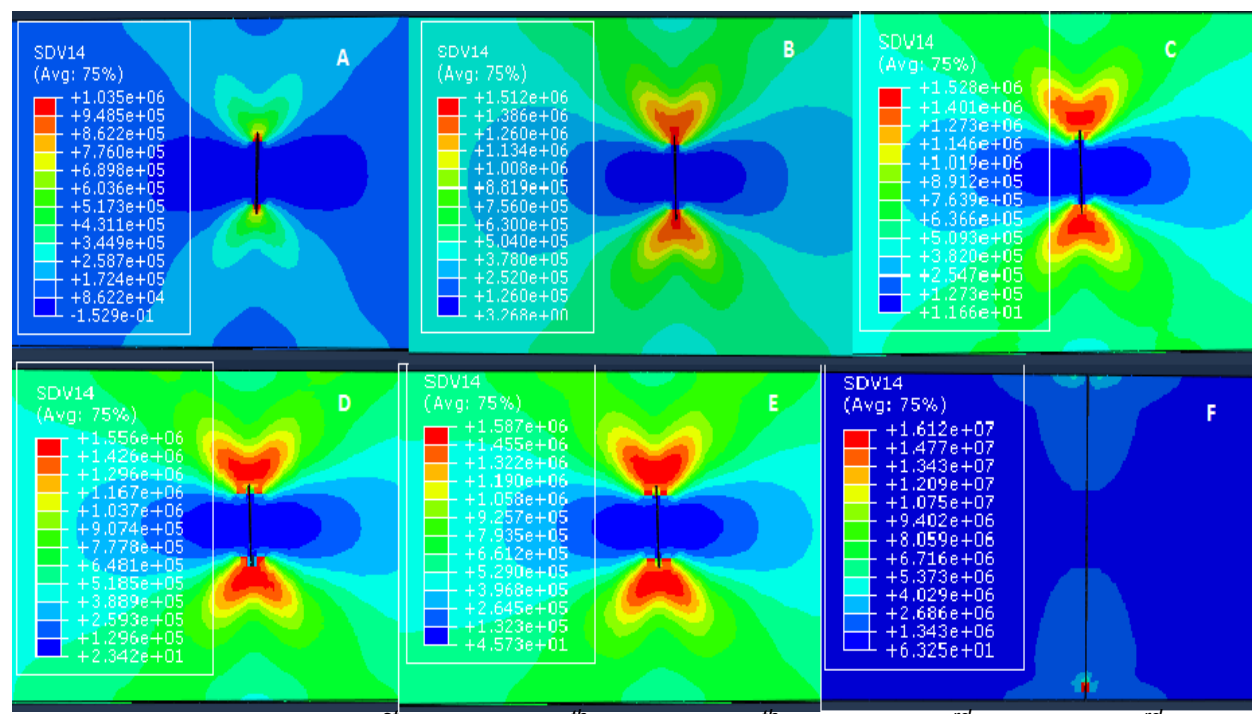

Fig. 8: Stored energy distributions: A) $1^{\text {st }}$ cycle, B) $15^{\text {th }}$ cycle, C) $25^{\text {th }}$ cycle, D) $33^{\text {rd }}$ cycle, E) $43^{\text {rd }}$ cycle, F) $49^{\text {th }}$ cycle

\section{Conclusions}

The theoretical and experimental study of energy balance in metals under plastic deformation and failure was carried out. The mathematical model describing the temperature evolution, heat dissipation and stored energy in deformed metals was proposed. These equations were adopted for the three-dimensional modeling in the finite-element package Simulia Abaqus 6.13 with the use of subroutine UMAT. The main advantage of this model is the possibility of calculation of the stored energy value which opens up possibilities for the development nondestructive damage monitoring techniques.

Efficiency of the model was demonstrated by the simulation of the energy balance in 304(L) austenitic stainless steel under quasi static deformation. Results of simulation have shown that the energy storage rate is the function of material deformation. The obtained results are in a quantitative agreement with the experimental data. The crack propagation process was simulated using XFEM capability in Abaqus and critical stored energy criterion was used for the crack propagation.

The experimental investigation of crack propagation process required the development both the data processing algorithms for temperature data and additional experimental setup for measurement of heat flux from the sample. It gives us an effective experimental method to study the energy dissipation during the mechanical experiments.

As a result of experimental program it was show that in the Paris regime the heat dissipation at the crack tip can be divided in regimes. The first regime is characterized by the constant value of heat dissipation form the crack tip. The second regime corresponds to the sharp rise of heat flux. The analysis of experimental data shows:

under the first regime the crack rate is linear function of crack length and power of heat dissipation $\left(\frac{\partial a_{1}}{\partial N} \sim Q_{1} a_{1}\right)$; under the second regime the crack rate is linear function power of heat dissipation $\left(\frac{\partial a_{2}}{\partial N} \sim Q_{2}\right)$.

These results were confirmed by the experiments with different materials and with different sample geometries.

\section{REFERENCES}

[1] lino Y. "Fatigue crack propagation work coefficient - a material constant giving degree of resistance to fatigue crack growth" Engineering fracture mechanics, vol. 12, pp. 279-299, 1979.

[2] Chow C. L., Lu T. J. "Cyclic J-integral in relation to fatigue crack initiation and propagation". Engineering Fracture Mechanics, vol. 39, Is. 1, pp. 1-20, 1991.

[3] Dowling N.E., Begley J.A. "Mechanics of crack growth". ASTM STP 590. Philadelphia, PA: American Society for Testing and Materials. pp. 83-104, 1976.

[4] Lindley T. C., McCartney L. N. "Mechanics and mechanisms of fatigue crack growth". Developments in Fracture Mechanics-2, Applied Science, 1981

[5] Izumi Y., Fine M. E., Mura T. "Energy considerations in fatigue crack propagation". Int. J. Fracture, vol. 17, pp. 15-25, 1981. 
[6] Chakrabarti A. K. "En energy-balance approach to the problem of fatigue-crack growth". Engng Fracture Mech., vol. 10, pp. 469-483, 1978.

[7] Christensen R. M., Wu E. M. "A theory of crack growth in viscoelastic materials". Engng Fracture Mech., vol. 14, pp. 215-225, 1981.

[8] Bodner S. R., Davidson D. L., Lankford J." A description of fatigue crack growth in terms of plastic work". Engng Fracture Mech., vol. 17, pp. 189-191, 1983.

[9] Short J. S., Hoeppner D. W. A "Global/local theory of fatigue crack propagation". Engineering Fracture mechanics, vol. 33, Is. 2, pp. 175-184, 1989.

[10] Chudnovsky A., Moet A.. "Thermodynamics of translational crack layer propagation". Journal of Materials Science, vol. 20, pp. 630-635, 1985.

[11] Strüwe A., Pippan R.. "On the energy balance of fatigue crack growth". Computers and Structures, vol. 44, Is. $1 / 2 .$, pp. 13-17, 1992.

[12] Izyumova A., Plekhov O. "Calculation of the energy J-integral in plastic zone ahead of a crack tip by infrared scanning". Fatigue and fracture of engineering materials and structures, vol. 37, issue 12, pp. 1330-1337, 2014.

[13] Ivanova V.S., Terentiev V.F. "The nature of the fatigue of metals". Moscow «Metallurgy», pp. 456, 1975 (In Russian)

[14] Troshchenko V.T. "Deformation and fracture of metals under high cyclic loading”. Kiev: «Naukova Dumka», p. 344, 1981 (In Russian)

[15] Fedorov V.V. "Thermodynamic aspects of strength and fracture of solids". Tashkent: «FAN» Uz SSR, p. 168, 1979. (In Russian)

[16] Aravas N., Kim K-S., Leckie F.A. "On the calculation of the stored energy of cold work". J. Eng. Mater-T, vol. 112, pp. 465-470, 1990.

[17] Szczepinski W. "The stored energy in metals and the concept of residual microstresses in plasticity". Arch. Mech, vol. 53, pp. 615-629, 2001.

[18] Oliferuk W., Maj M., Stress-strain curve and stored energy during uniaxial deformation of polycrystals, Eur. J. Mech. A-Solids. 28 (2009) pp. 266-272.

[19] Benzerga A. A., et al., "The stored energy of cold work: predictions from discrete dislocation plasticity". Acta Mater., vol.53, pp. 4765-4779, 2005.

[20] Rosakis P., et al., "A thermodynamic internal variable model for the partition of plastic work into heat and stored energy in metals". J. Mech. Phys. Solids, vol. 48, pp. 581-607, 2000.

[21] Chaboche J-L. "Cyclic viscoplastic constitutive equations". J. Appl. Mech., vol. 60, pp. 813-828, 1993.

[22] Naimark O.B. "Collective properties of defects ensemble and some nonlinear problems of plasticity and failure". Phys. Mesomech., vol. 50, pp.127-136, 2009.

[23] Oliferuk W., Maj M. "Energy storage rate in non-homogeneous deformation". Proceedings of the $21^{\text {st }}$ International congress of theoretical and applied mechanics (ICTAM04). E-book, 1185, 2005. 